\title{
Nuclear Magnetic Resonance Parameters of Pure and Diborinin-Doped (6,0) Single-Walled Zigzag BNNT: DFT Study
}

\author{
Sattar Arshadi, Saboora Abedini, Ameneh Asghari, and Fatemeh Alipour Zaghmarzi \\ Department of Chemistry, Payame Noor University, Tehran 19395-4697, Iran \\ Correspondence should be addressed to Sattar Arshadi, chemistry_arshadi@yahoo.com
}

Received 24 June 2012; Revised 14 September 2012; Accepted 4 October 2012

Academic Editor: Sebastian O. Danielache

Copyright (c) 2013 Sattar Arshadi et al. This is an open access article distributed under the Creative Commons Attribution License, which permits unrestricted use, distribution, and reproduction in any medium, provided the original work is properly cited.

\begin{abstract}
A computational study on the basis of density functional theory (DFT) calculations has been performed to investigate the properties of the electronic structure of $(6,0)$ zigzag boron nitride nanotubes and two models $((a)$ and $(b))$ of diborinin-doped boron nitride nanotubes (DBD-BNNTs). The calculated structural energies yield similar values for two models of DBD-BNNTs. Isotropic (CS $\left.{ }^{I}\right)$ and anisotropic $\left(\mathrm{CS}^{A}\right)$ chemical shielding parameters of the optimized BNNT and DBD-BNNTs are calculated. The results illustrate that the changes in chemical shielding tensors of ${ }^{11} \mathrm{~B}$ and ${ }^{15} \mathrm{~N}$ nuclei are more significant in the nearest neighborhood of the diborinin ring due to doping process. The changes of the electronic sites of the $\mathrm{N}$ atoms are also more significant than those of the $\mathrm{B}$ atoms. The dipole moments of the diborinin-doped BNNT structures show changes with respect to the pristine model. It is clear that the doping of diborinin ring decreases the energy gaps of the pure BNNT. For the pure model, the HOMO is located on the nitrogen atoms, and the LUMO is uniformly distributed throughout the B-N bonds. In contrast, for the diborinin-doped models, the majority of the HOMO and LUMO are located at the diborinin-doped regions.
\end{abstract}

\section{Introduction}

Nanostructures such as nanotube are currently the subject of intense research because of the potential applications in nanoscale electronic and optoelectronic fields [1-4]. After the exploration of carbon nanotubes (CNT) by Iijima in 1991 [1], nanotubes of other chemical compositions such as boron nitride have also been investigated [5]. Boron nitride nanotubes (BNNTs) have been the focus of several experimental and theoretical studies on account of their potential applications in high speed electronics [6]. BNNTs were successfully synthesized [7] shortly after prediction in theory [8].

BNNTs are a typical member of III-V, compound with morphology similar to that of carbon nanotubes (CNTs) but with their own distinct properties. However, the electrical properties of BNNTs are very different from those of CNTs. While CNTs come in three basic forms (metallic, smallgap semiconductor, and modest-gap semiconductor) rely on diameter and chirality, first approximation of BNNTs are electrically uniform with an energy gap of about $4-5 \mathrm{eV}$ [9]. This energy gap is independent of wall number, diameter, or chirality. For low applied electric fields, BNNTs are thus essentially insulating.

A tubular structure of $\mathrm{BN}$ can be formed by rolling up a sheet of hexagonal rings with boron and nitrogen in equal proportions possessing peculiar electrical [10], optical [11], and thermal [12] properties, which drastically differ from those of CNTs. BNNTs have high surface area, unique physical properties and morphology. On top of that, BNNTs possess high chemical stability. These properties make BNNTs one of most promising materials for nanotechnology applications, especially under oxidative, hazardous and hightemperature environments. But there have been quite a few attempts to control the properties by incorporating extrinsic foreign atom doping. These substitutions in BNNT significantly modify the chemical binding configuration, physical, chemical, and optical properties in comparison to pure BNNT [13].

Carbon-, Si-, Ti- and Pt-doped [14-18] BNNTs have been studied, and their results reveal that the electronic properties of BNNTs might be exploited for various applications. Generally, doping of BNNTs with other elements is a promising approach to control their electronic structures. 


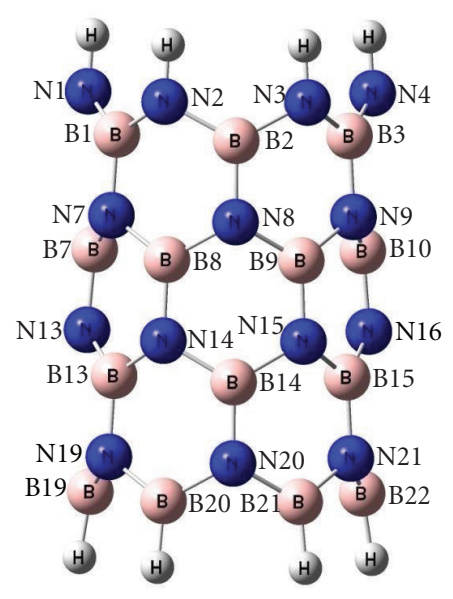

(a)

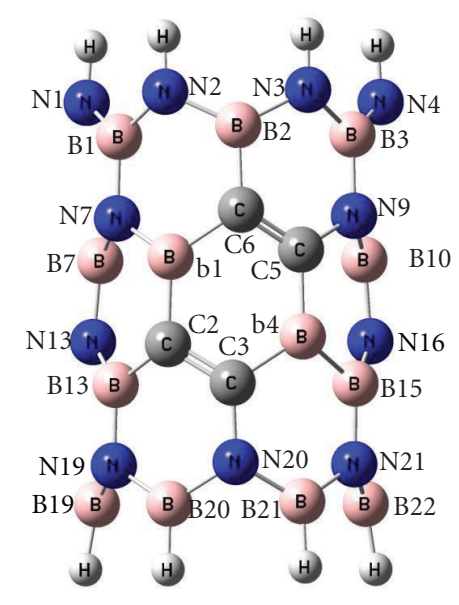

(c)

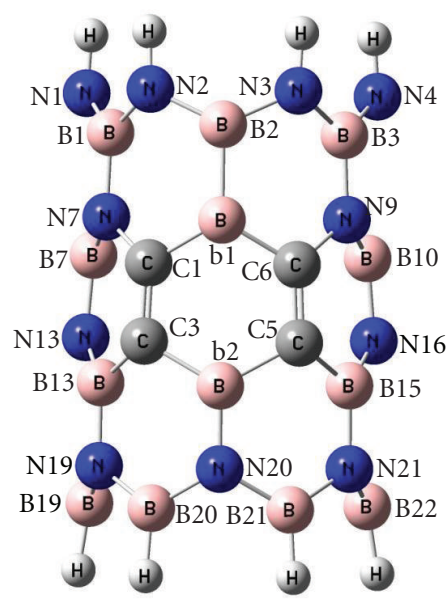

(b)

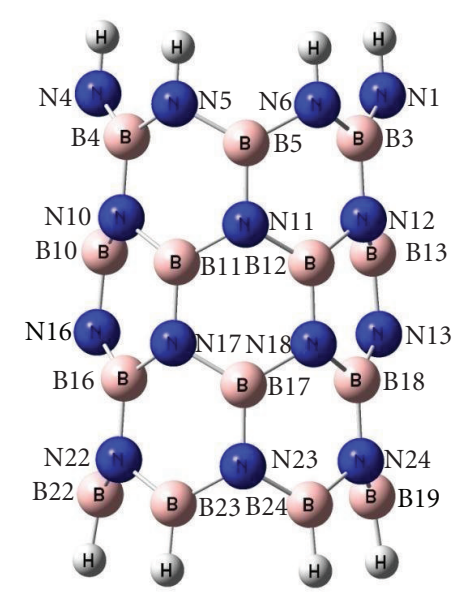

(d)

FIgURE 1: Typical geometric structures pure and diborinin-doped $(6,0)$ single-walled zigzag BNNT; (a) front side of pure model, (b) and (c) front side of DBD-BNNTs, and (d) back side of pure and DBD-BNNTs models.

On the other hand, the BNNTs are produced through heating of $\mathrm{C}$ templates with $\mathrm{B}_{2} \mathrm{O}_{3}$ in a flowing $\mathrm{N}_{2}$ atmosphere at $1503-1773 \mathrm{~K}$. Due to this process, carbon atoms replace with $\mathrm{B}$ and $\mathrm{N}$ atoms but somewhere, diborinin ring formation as a defection in BNNT structure come into view.

Exploitation of the chemical and physical properties of nanotubes requires understanding and control of their structure during synthesis. While several techniques are used to probe nanotube structure, full characterization is not possible. ${ }^{11} \mathrm{~B}$ and ${ }^{15} \mathrm{~N}$ NMR spectroscopy have the potential to provide a more detailed structural characterization.

In this direction, we have studied the structure of electronic properties and nuclear magnetic resonance (NMR) parameters of ${ }^{11} \mathrm{~B}$ and ${ }^{15} \mathrm{~N}$ nuclei for pure $(6,0)$ singlewalled zigzag BNNT ((a) model) and DBD-BNNT ((b) and (c) models) via density functional theory method (DFT).
The zigzag $(6,0)$ BNNT is chosen here because the zigzag orientation is a preferred growth one and the $(6,0)$ BNNT has a moderate diameter. In addition, we have investigated atomic charge of distribution in nanotubes by natural bond orbital (NBO).

\section{Models and Computational Details}

DFT calculations were carried out using the Gaussian 98 program [19] on the structure of pure and DBD-BNNT BNNTs. DFT has become extremely popular for molecular applications because it accounts for the correlation energy in computationally efficient manner and offers a substantially improved accuracy over conventional approaches. Subsequently, frequency calculations (keyword: FREQ = NORAMAN) were carried out by standard techniques on 
the optimized structures. For minimum state species, only real frequency values (with a positive sign) are accepted.

As was pointed out in the previous section, calculations have been performed on (a), (b), and (c) models (Figure 1). Two structural isomers of DBD-BNNTs ((b) and (c) models) were considered in the calculations within four $\mathrm{sp}^{2}$ hybridized carbon and two borons atoms (diborinin ring) were substituted instead of three boron and three nitrogen atoms in the middle of the nanotube.

At the first step of this study, each of the considered pure and diborinin-doped representative models of BNNTs was allowed to fully relax during the geometrical optimization and frequency calculation. The geometries were optimized at the B3LYP/6-311G** level of theory. Subsequently, the $\mathrm{NBO}$ analysis was carried out on the B3LYP/6-311G** wave functions. From the NBO calculations, highest-occupied molecular orbital (HOMO) and lowest-unoccupied molecular orbital (LUMO) energies, the band gap energies, dipole moments and electronegativity $(\chi)$ for $(a),(b)$, and (c) models of BNNTs were obtained.

Also, we investigated electronegativity $(\chi)$ in the optimized structures. Within the conceptual framework of DFT, the electronegativity of a system of $N$ particles, with total energy $E$ and subject to the external potential $v(r)$, is defined as

$$
\chi=-\left(\frac{\partial E}{\partial N}\right)_{v(r), T}
$$

In (1), $\chi$ is the electronegativity [20-25]. Pauling introduced the concept of electronegativity as the power of an atom in a molecule to attract electrons [20]. Using the method of finite difference, an operational and approaching definition of $\chi$ is:

$$
\chi=\frac{1}{2}(\mathrm{IP}+\mathrm{EA})
$$

where IP is the ionization potential and EA is the electron affinity of the system. Within the validity of Koopman's theorem for closed-shell species, the frontier orbital energies are given by

$$
-E_{\mathrm{LUMO}}=I P ; \quad-E_{\mathrm{HOMO}}=E A .
$$

Therefore, on the basis of orbitals, we can write

$$
\chi=-\frac{1}{2}\left(E_{\mathrm{LUMO}}+E_{\mathrm{HOMO}}\right) .
$$

$E_{\mathrm{LUMO}}$ and $E_{\mathrm{HOMO}}$ are the energies of the lowest unoccupied and the highest occupied molecular orbitals LUMO and HOMO, respectively.

Finally nuclear magnetic resonance (NMR) parameters at ${ }^{11} \mathrm{~B}$ and ${ }^{15} \mathrm{~N}$ nuclei of the optimized structures have been calculated. As noted, we have carried out these calculations at the GIAO/B3LYP/6-311++G ${ }^{* *}$ level, one of the best compromises between accuracy and computer time [26].

Mathematically, the anisotropic and isotropic chemical shifts are described by a 3 by 3 matrix. In this principal axis system, the chemical shift tensor is fully described by the three diagonal elements - the principal components $\left(\sigma_{33}>\right.$ $\left.\sigma_{22}>\sigma_{11}\right)$-and the three eigenvectors or Euler angles describe the orientation of the principal axes with respect to an arbitrary frame. Therefore, (5) and (6) are used to convert the calculated chemical shielding (CS) tensors to the isotropic $\left(\mathrm{CS}^{I}\right)$ and anisotropic $\left(\mathrm{CS}^{A}\right)$ parameters. The isotropic value (the centre of gravity) is the average value of the principal components, and the anisotropy describe the largest separation from the centre of gravity. The evaluated NMR parameters for (b) and (c) models of DBD-BNNTs are listed in Tables 2 and 3, respectively. For the ease of comparison, the NMR parameters for (a) model of pure BNNT are listed in these tables. The following are equations (5) and (6):

$$
\begin{aligned}
\operatorname{CS}^{I}(\mathrm{ppm}) & =\frac{\left(\sigma_{11}+\sigma_{22}+\sigma_{33}\right)}{3}, \\
\operatorname{CS}^{A}(\mathrm{ppm}) & =\frac{\sigma_{33}-\left(\sigma_{11}+\sigma_{22}\right)}{2} .
\end{aligned}
$$

\section{Result and Discussion}

3.1. Geometries. As shown in Figure 1, by substituting three $\mathrm{B}$ and three $\mathrm{N}$ atoms of pure BNNT ((a) model) with four $\mathrm{sp}^{2}$ hybridized carbons and two boron atoms, in the middle of the nanotube, (b) and (c) models of DBD-BNNTs are produced.

At the first step, geometrical optimizations and frequency calculations have been successfully carried out for the pure and doped models $(\mathrm{a}, \mathrm{b}$, and $\mathrm{c}$ ) of single-wall $(6,0)$ BNNT at the level of B3LYP/6-311G** basis set. The optimized geometries of pure and doped models of the BNNTs are listed in Figures 2 and 3. These figures present the optimized parameters including the bond lengths and bond angles for three models that investigated $(6,0) \mathrm{BNNT}$. In the pure BNNT (Figure 2(a)), the average B-N bond length is $1.46 \AA$, consistent with previously reported DFT calculations [27, 28]. This value was also changed by the diborinin-doping in the DBD-BNNT due to existence of $\mathrm{B}-\mathrm{C}, \mathrm{N}-\mathrm{C}, \mathrm{B}-\mathrm{B}$, and $\mathrm{C}=\mathrm{C}$ bonds, in addition to, $\mathrm{B}-\mathrm{N}$ one.

However, the effects of this deformation are consequential, just at the geometrical properties of the nearest atoms to the diborinin-doped ring, whereas those of other atoms are almost remained unchanged. As shown in Figures 2(b) and $2(\mathrm{c})$, the average $\mathrm{C}=\mathrm{C}$ and $\mathrm{B}-\mathrm{C}$ bond lengths $(1.36 \AA$ and $1.56 \AA$, resp.) are different from $\mathrm{B}-\mathrm{N}$ bond length $(1.46 \AA)$. Moreover, the changes of the bond angles are more than those of the bond lengths in the $(6,0)$ zigzag DBD-BNNT (Figures 2 and 3 ).

The calculated structural energies, LUMO-HOMO gaps, electronegativity $(\chi)$, and dipole moments for three models of the investigated BNNTs have been listed in Table 1.

The calculated structural energies of two structural isomers ((b) and (c) models) of DBD-BNNT are equal to $-51.2 \mathrm{keV}$ and indicate no difference between them.

For better understanding of the nature of interaction in the diborinin-doped models, we studied the electronic properties of the models. The energies of both the highest occupied molecular orbital (HOMO) and the lowest unoccupied molecular orbital (LUMO) are negative in all of 
TABLE 1: B3LYP/6-311G** calculated properties of pure and diborinin-doped ((b) and (c) models) of BNNT.

\begin{tabular}{lcccccc}
\hline Systems & HOMO $(\mathrm{eV})$ & LUMO $(\mathrm{eV})$ & $\Delta E_{(\text {LUMO-HOMO })}(\mathrm{eV})$ & $\chi(\mathrm{eV})$ & Dipole moment $($ Debye $)$ & Energy $(\mathrm{keV})$ \\
\hline Pure (a) & -6.86 & -1.99 & 4.87 & 4.42 & 7.47 & -52.2 \\
DBD-BNNT (b) & -5.93 & -3.28 & 2.64 & 4.61 & 9.28 & -51.2 \\
DBD-BNNT (c) & -5.99 & -3.15 & 2.83 & 4.57 & 6.98 & -51.2 \\
\hline
\end{tabular}

TABLE 2: The $\mathrm{CS}^{I}$ and $\mathrm{CS}^{A}$ parameters of the (b) model of DBD-BNNT.

\begin{tabular}{|c|c|c|c|c|c|c|c|c|c|c|c|}
\hline \multicolumn{6}{|c|}{$\mathrm{CS}^{I}$ (ppm) } & \multicolumn{6}{|c|}{$\mathrm{CS}^{A}(\mathrm{ppm})$} \\
\hline${ }^{11} \mathrm{~B}$ & Pure & DBD-BNNT & ${ }^{15} \mathrm{~N}$ & Pure & DBD-BNNT & ${ }^{11} \mathrm{~B}$ & Pure & DBD-BNNT & ${ }^{15} \mathrm{~N}$ & Pure & DBD-BNNT \\
\hline layer 1 & & & & & & layer 1 & & & & & \\
\hline B1 & & 72.5 & N1 & & 144.1 & B1 & & 43.2 & N1 & & 90.8 \\
\hline B2 & & 67.2 & $\mathrm{~N} 2$ & & 127.3 & B2 & & 80.4 & $\mathrm{~N} 2$ & & 124.7 \\
\hline B3 & 705 & 72.6 & N3 & 147.5 & 128.1 & B3 & 44.5 & 42.9 & N3 & 95 & 123.3 \\
\hline B4 & 10.0 & 70.8 & $\mathrm{~N} 4$ & $14 \% .0$ & 144.1 & B4 & $44 . J$ & 44.4 & N4 & (3) & 91.4 \\
\hline B5 & & 69.8 & N5 & & 147.6 & B5 & & 44.7 & N5 & & 95.3 \\
\hline B6 & & 70.9 & N6 & & 146.9 & B6 & & 44.2 & N6 & & 96.6 \\
\hline layer 2 & & & & & & layer 2 & & & & & \\
\hline B7 & & 67.3 & N7 & & 80.3 & B7 & & 43.0 & N7 & & 213.0 \\
\hline B8 & & - & N8 & & - & B8 & & - & N8 & & - \\
\hline B9 & 69 & - & N9 & 114 & 80.0 & B9 & 405 & - & N9 & 187 & 213.2 \\
\hline B10 & (3) & 67.4 & N10 & 114 & 116.4 & $\mathrm{~B} 10$ & 40.3 & 43.7 & N10 & 107 & 187.0 \\
\hline B11 & & 68.7 & N11 & & 113.8 & B11 & & 39.0 & N11 & & 187.9 \\
\hline B12 & & 68.3 & N12 & & 115.8 & B12 & & 40.7 & N12 & & 187.5 \\
\hline layer 3 & & & & & & layer 3 & & & & & \\
\hline B13 & & 67.2 & N13 & & 94.4 & B13 & & 59.5 & N13 & & 232.0 \\
\hline B14 & & - & N14 & & - & B14 & & - & N14 & & - \\
\hline B15 & 695 & 66.9 & N15 & 111 & - & B15 & 43 & 60.3 & N15 & 1955 & - \\
\hline B16 & 09.0 & 69.4 & N16 & 111 & 94.0 & B16 & $4 J$ & 42.5 & N16 & 193.0 & 232.6 \\
\hline B17 & & 68.9 & N17 & & 109.7 & B17 & & 43.6 & N17 & & 197.4 \\
\hline B18 & & 69.3 & N18 & & 110.5 & B18 & & 42.8 & N18 & & 197.0 \\
\hline layer 4 & & & & & & layer 4 & & & & & \\
\hline B19 & & 64.9 & N19 & & 67.9 & B19 & & 60.8 & N19 & & 257.2 \\
\hline B20 & & 63.1 & N20 & & 49.1 & B20 & & 59.8 & N20 & & 267.0 \\
\hline B21 & 64 & 63.6 & N21 & 75 & 67.5 & B21 & 60 & 58.5 & N21 & 2415 & 258.0 \\
\hline B22 & 04 & 65.0 & N22 & 13 & 73.9 & B22 & 00 & 60.5 & N22 & $241 . J$ & 243.3 \\
\hline B23 & & 63.8 & N23 & & 75.5 & B23 & & 59.5 & N23 & & 242.2 \\
\hline B24 & & 63.9 & N24 & & 73.5 & B24 & & 58.9 & N24 & & 243.2 \\
\hline
\end{tabular}

model systems under study. The calculated energy gap for the pure BNNT is $4.87 \mathrm{eV}$ (Table 1 ). In comparison to the pure systems, the energy gaps between HOMO and LUMO, $\left(\Delta E_{\text {(LUMO-HOMO) }}\right)$, have small values in (b) and (c) models of DBD-BNNT. The electronic densities of states (DOSs) of pure and diborinin-doped BNNT are plotted in Figure 4 for better understanding, the effect of diborinin ring doping to the electronic structure of BNNT. The electronic structures observed near Fermi level of the pure BNNT (Figure 4(a)) also show significant changes due to the diborinin ring doping (see Figures 4(b)-4(c)). So, the band gaps near Fermi level become sharply narrower. It is clear that the doping of diborinin ring decreases the energy gaps of the pure BNNT.

The HOMO and LUMO, the pure and diborinin-doped models are plotted in Figure 5. For the pure model, the
HOMO is located on the nitrogen atoms of the $(6,0)$ zigzag BNNT model and corresponds to the lone pair of electron on nitrogen atoms. In contrast, the LUMO is uniformly distributed throughout the $\mathrm{B}-\mathrm{N}$ bonds. For the diborinindoped models, the majority of the HOMO and LUMO are located at the diborinin-doped regions.

The edges of the armchair BNNTs include N and B atoms in both ends. In contrast to armchair models, edges of the zigzag BNNTs consist of one type atoms, either N or B atoms; if one of the ends is formed by $\mathrm{N}$ atoms, the other end is formed by B atoms. The B atoms are slightly positive and the $\mathrm{N}$ atoms are slightly negative. Therefore, the zigzag BNNT is a polar material (has a nonzero dipole moment) and this entry could be better seen by the value of its dipole moment. Comparing the values of the dipole moments for the two 
TABLE 3: The $\mathrm{CS}^{I}$ and $\mathrm{CS}^{A}$ parameters of the (c) model of DBD-BNNT.

\begin{tabular}{|c|c|c|c|c|c|c|c|c|c|c|c|}
\hline \multicolumn{6}{|c|}{$\mathrm{CS}^{I}(\mathrm{ppm})$} & \multicolumn{6}{|c|}{$\mathrm{CS}^{A}(\mathrm{ppm})$} \\
\hline${ }^{11} \mathrm{~B}$ & Pure & DBD-BNNT & ${ }^{15} \mathrm{~N}$ & Pure & DBD-BNNT & ${ }^{11} \mathrm{~B}$ & Pure & DBD-BNNT & ${ }^{15} \mathrm{~N}$ & Pure & DBD-BNNT \\
\hline layer 1 & & & & & & layer 1 & & & & & \\
\hline B1 & & 68.6 & N1 & & 143.3 & B1 & & 46.2 & N1 & & 93.2 \\
\hline B2 & & 65.1 & N2 & & 137.6 & B2 & & 63.0 & N2 & & 102.9 \\
\hline B3 & 705 & 68.6 & N3 & 1475 & 130.3 & B3 & 445 & 48.8 & N3 & 95 & 129.6 \\
\hline B4 & 10.0 & 69.5 & $\mathrm{~N} 4$ & 147.0 & 148.0 & B4 & TI.J & 46.4 & N4 & (נ) & 92.8 \\
\hline B5 & & 70.1 & N5 & & 148.4 & B5 & & 45.8 & N5 & & 92.5 \\
\hline B6 & & 69.1 & N6 & & 147.8 & B6 & & 47.5 & N6 & & 92.4 \\
\hline layer 2 & & & & & & layer 2 & & & & & \\
\hline B7 & & 68.5 & N7 & & 98.2 & B7 & & 39.7 & N7 & & 211.8 \\
\hline B8 & & - & N8 & & - & B8 & & - & N8 & & - \\
\hline B9 & 69 & - & N9 & 114 & 86.3 & B9 & 405 & - & N9 & 187 & 184.3 \\
\hline B10 & (0) & 67.9 & N10 & 114 & 110.6 & $\mathrm{~B} 10$ & T0.J & 39.0 & N10 & 107 & 184.0 \\
\hline B11 & & 67.9 & N11 & & 111.7 & B11 & & 41.1 & N11 & & 190.3 \\
\hline B12 & & 67.9 & N12 & & 113.6 & B12 & & 42.1 & $\mathrm{~N} 12$ & & 185.5 \\
\hline layer 3 & & & & & & layer 3 & & & & & \\
\hline B13 & & 66.3 & N13 & & 108.0 & B13 & & 64.1 & N13 & & 205.6 \\
\hline B14 & & - & N14 & & - & B14 & & - & N14 & & - \\
\hline B15 & 695 & 56.6 & N15 & 111 & - & B15 & 43 & 89.9 & N15 & 1955 & - \\
\hline B16 & 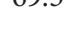 & 70.3 & N16 & 111 & 84.8 & B16 & $7 J$ & 43.8 & N16 & נ. & 243.9 \\
\hline B17 & & 68.8 & N17 & & 112.3 & B17 & & 43.8 & N17 & & 196.5 \\
\hline B18 & & 69.2 & N18 & & 111.4 & B18 & & 43.0 & N18 & & 195.9 \\
\hline layer 4 & & & & & & layer 4 & & & & & \\
\hline B19 & & 65.0 & N19 & & 67.9 & B19 & & 58.3 & N19 & & 276.7 \\
\hline B20 & & 69.8 & N20 & & 45.9 & B20 & & 60.0 & N20 & & 256.6 \\
\hline B21 & 64 & 66.0 & N21 & 75 & 65.8 & B21 & 60 & 55.4 & N21 & 2415 & 273.4 \\
\hline B22 & & 66.0 & N22 & (J) & 70.7 & B22 & & 59.5 & N22 & & 247.9 \\
\hline B23 & & 64.5 & N23 & & 73.8 & B23 & & 58.1 & N23 & & 244.5 \\
\hline B24 & & 64.9 & N24 & & 70.4 & B24 & & 58.1 & N24 & & 247.4 \\
\hline
\end{tabular}

diborinin-doped models indicate, that the value for the (b) model is significantly increased ( $D=9.28$ Debye) whereas for (c) model is significantly reduced ( $\mathrm{D}=6.98$ Debye), compared with the pure model $(\mathrm{D}=7.47$ Debye), which means that the orientations of diborinin ring in two diborinindoped models foundation diverse dipole moment for these models (Figure 1 and Table 1).

3.2. NMR Parameter. NMR spectroscopy has proven to be an exceptionally powerful technique in characterizing molecular systems and structures. So, we have performed theoretical calculations of ${ }^{11} \mathrm{~B}$ and ${ }^{15} \mathrm{~N}$ NMR data for (a), (b), and (c) models to study the influence of diborinin ring doping on the electronic structure properties of the $(6,0)$ BNNT.

3.2.1. Pure Model. Tables 2 and 3 present the calculated NMR parameters for various ${ }^{11} \mathrm{~B}$ and ${ }^{15} \mathrm{~N}$ nuclei in the pure (a) model of BNNT. This model includes $24 \mathrm{~B}$ and $24 \mathrm{~N}$ atoms, where the edges are saturated by $12 \mathrm{H}$ atoms (Figure 1 ). The edge of this model consists of one type atom, either $\mathrm{N}$ or $\mathrm{B}$ atoms; if one of the end is formed by $\mathrm{N}$ atoms (N-tip), the other ends is formed by B atoms (B-tip).
According to the previous studies, to compare CS tensors between pure and doped models, ${ }^{11} \mathrm{~B}$ and ${ }^{15} \mathrm{~N}$ nuclei in the pure (a) model are divided into four different layers which sense similar electrostatic properties in each layer, yielding the same values of the $\mathrm{CS}^{I}$ and $\mathrm{CS}^{A}$ parameters [29]. Almost no significant difference is observed in the calculated NMR parameters for the atoms of each layer; hence, just the average values in each layer for ${ }^{15} \mathrm{~N}$ and ${ }^{11} \mathrm{~B}$ nuclei are reported in Tables 2 and 3.

The first layer consists of N1-N6 atoms and the last layer contains B19-B24 atoms, which forms the N-tip and B-tip of the considered models of BNNT, respectively. The values of NMR properties for the ${ }^{11} \mathrm{~B}$ and ${ }^{15} \mathrm{~N}$ nuclei of the pure (a) model indicate that the B-tip has the smallest value of $\mathrm{CS}^{I}$ for both ${ }^{11} \mathrm{~B}(64 \mathrm{ppm})$ and ${ }^{15} \mathrm{~N}(75 \mathrm{ppm})$ nuclei. Similarly N-tip has the smallest value of $\mathrm{CS}^{A}$ for both ${ }^{11} \mathrm{~B}(44 \mathrm{ppm})$ and ${ }^{15} \mathrm{~N}$ (95 ppm) nuclei, among the other layers of nuclei.

The nature of the valence shells of the $\mathrm{N}$ atoms, which have lone pair of electrons, and the $\mathrm{B}$ atoms, which have a lack of electrons, is different. Therefore, different behaviors of the CS properties are expected and are also observed for these atoms. For these reasons, the average values of $\mathrm{CS}^{I}$ are greater 


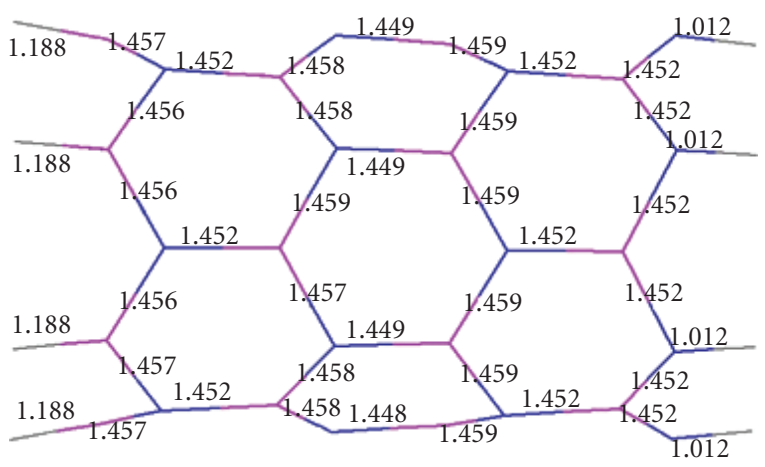

(a)

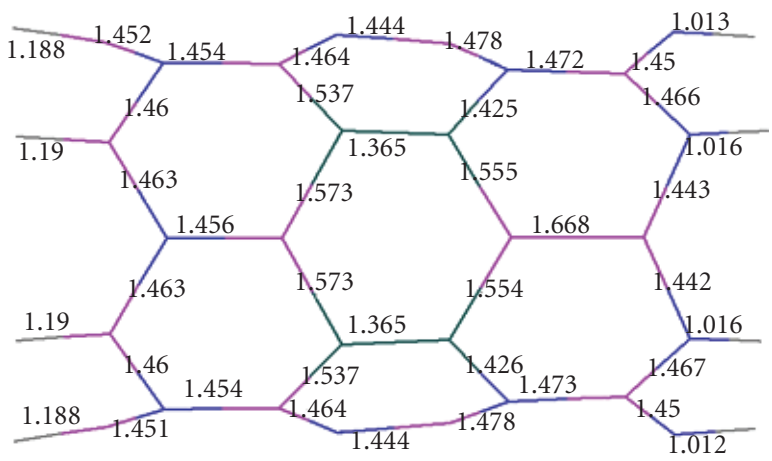

(b)

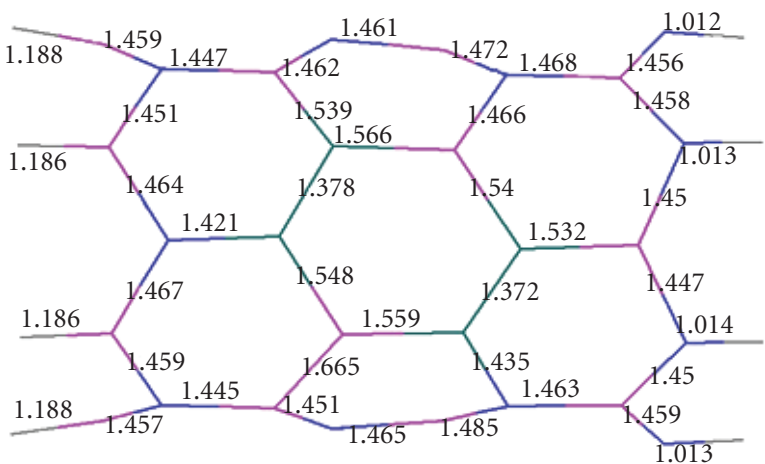

(c)

Figure 2: Optimized bond lengths ( $\AA$ ) at B3LYP/6-311G ${ }^{* *}$ level; (a) pure model, (b) and (c) diborinin-doped models.

than $\mathrm{CS}^{A}$ in ${ }^{11} \mathrm{~B}$ nuclei whereas the opposite order have been seen for ${ }^{15} \mathrm{~N}$ nuclei.

3.2.2. Diborinin-Doped Models. We characterize ${ }^{11} \mathrm{~B}$ and ${ }^{15} \mathrm{~N}$ NMR parameters of BNNT and illustrate how the diborinindoping modifies the NMR parameter of the pure BNNT. According to Gauge-Independent Atomic Orbital (GIAO) calculations performed later, doping of diborinin ring in $(6,0)$ BNNT, in both cases of doped models induces significant deformation of the $\mathrm{B}$ and $\mathrm{N}$ layers. These impurities lead to a significant change of the NMR parameter.

Compared to the pure model, in (b) model of the diborinin-doped (Figure 1(b)), N8, N14, N15, B8, B14 and B9 atoms are, respectively, doped by the b1, C3, C5, C2, b4

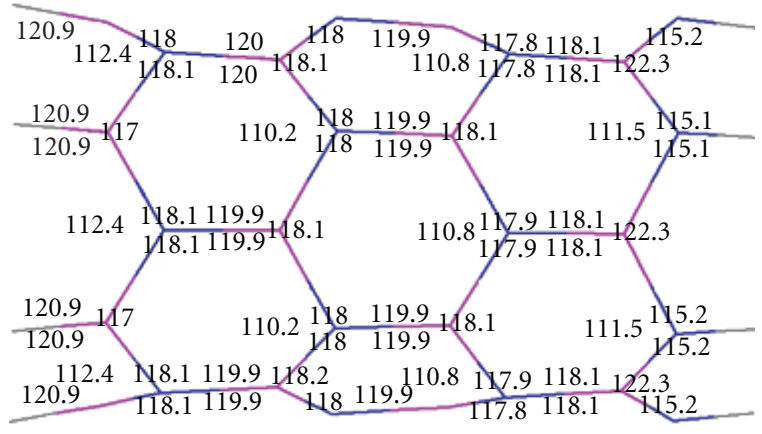

(a)

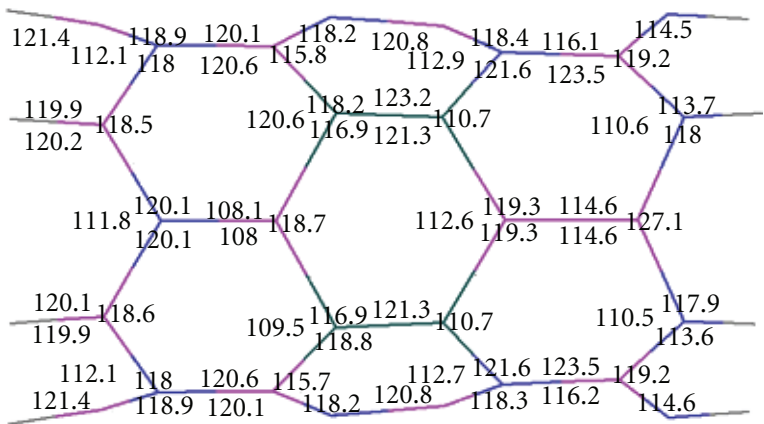

(b)

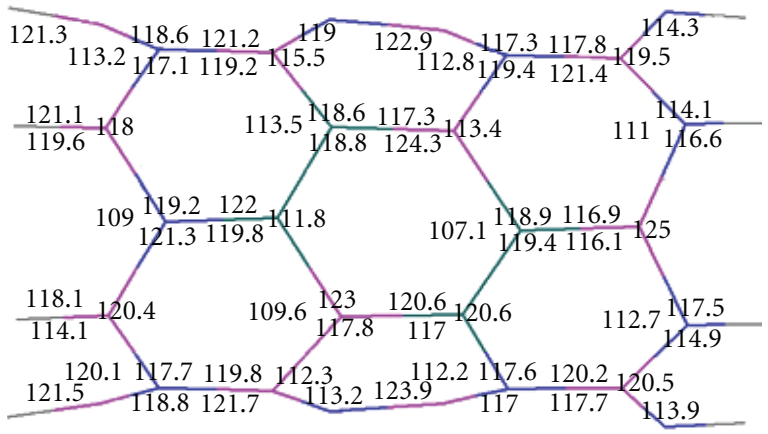

(c)

Figure 3: Optimized bond angles $\left({ }^{\circ}\right)$ at B3LYP/6-311G ${ }^{* *}$ level; (a) pure model, (b) and (c) diborinin-doped models.

and C6 atoms. In contrast of (b) model, in (c) model of the diborinin-doped (Figure 1(c)), N8, N14, N15, B8, B14 and B9 atoms, respectively, are doped by the C6, C2, B4, B1, C3 and $\mathrm{C} 5$ atoms.

Among the B atoms of the (b) and (c) models, B2, B13 and $\mathrm{B} 15$ are significantly influenced by diborinin-doping due to the (b) model B2, B13 and B15 are directly bonded to the b1, C3 and C5 atoms, respectively while in the (c) model, $\mathrm{B} 2, \mathrm{~B} 13$ and $\mathrm{B} 15$ are directly bonded to the $\mathrm{C} 6, \mathrm{C} 2$ and $\mathrm{b} 4$ atoms respectively, hence, their CS parameters detect some changes due to the diborinin doping. This observation was more significant for N7, N9, and N20 atoms which were directly bonded to the diborinin ring. This is because, in the DBD-BNNT, the CS parameters are proportional to the electronic density at the atomic sites and could detect any perturbation to these sites. 


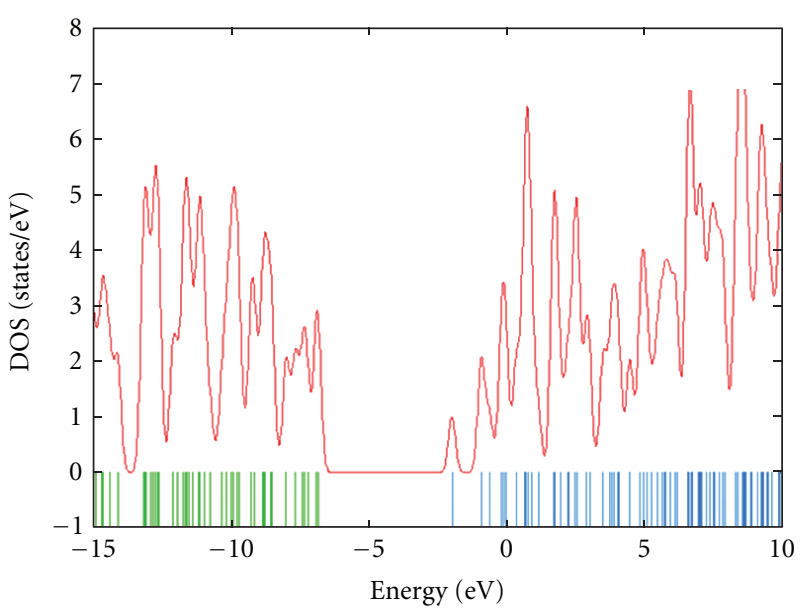

(a)

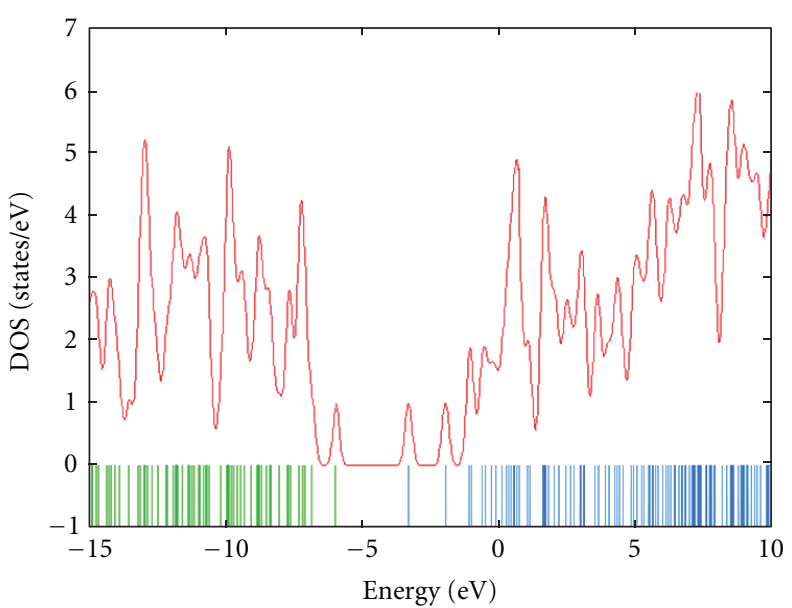

(b)

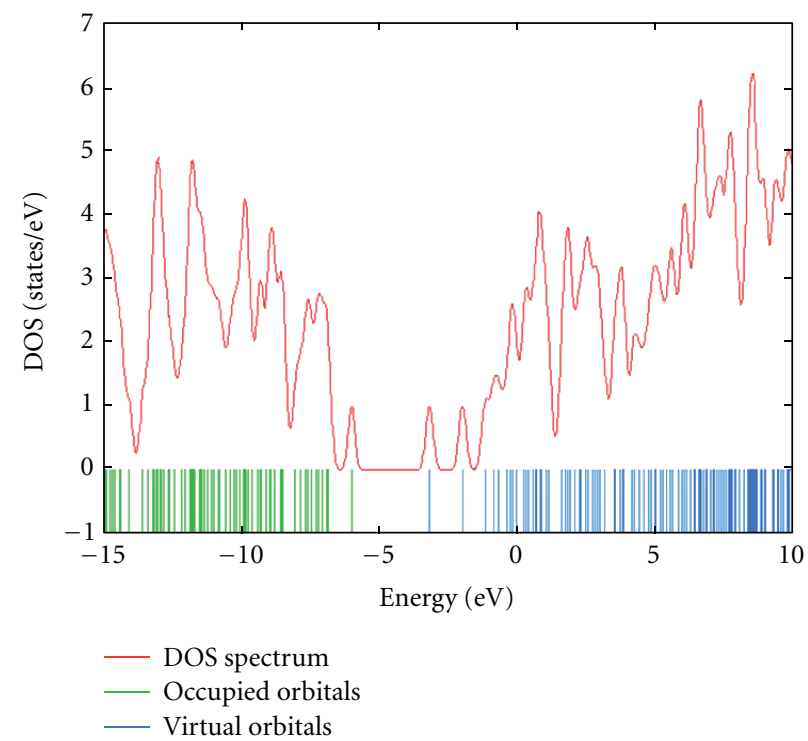

(c)

FIgURE 4: The density of states (DOS) of (a) pure model, (b) and (c) diborinin-doped models.

The results of Tables 2 and 3 reveal the significant effect of diborinin-doping at the sites of those ${ }^{11} \mathrm{~B}$ and ${ }^{15} \mathrm{~N}$ nuclei located in the nearest neighborhood (closer distance) of the DBD-BNNTs ((b) and (c) models), but negligible influence at the sites of those nuclei farther from the diborinin-doped rings. Also, in (b) and (c) models, the $\mathrm{CS}^{I}$ parameters are changed by diborinin-doping, the magnitude of the change for ${ }^{15} \mathrm{~N}$ being more significant rather than that for the ${ }^{11} \mathrm{~B}$ nuclei.

The $\mathrm{CS}^{I}$ parameters of B20 and B21 which were located in the B-tip of the (c) model are influenced indirectly by the diborinin-doping, whereas those of B20 and B21 in the (b) model remained unchanged.

Meanwhile, the magnitude of changes of the CS tensors at the sites of ${ }^{15} \mathrm{~N}$ nuclei was placed in the nearest neighborhood of diborinin ring is larger for the form (b) than the form (c), whereas the magnitude of changes of the CS tensors at the sites of ${ }^{15} \mathrm{~N}$ nuclei was placed in the first neighborhood of diborinin ring is larger for the form (c) than the form (b).

In the diborinin-doped models, when compared to the pure model, changes are greater in the values of CS parameters of those $\mathrm{N}$ atoms (N7, N9 and N20) which are directly bonded to diborinin ring in the (b) model borininthan in the (c) model whereas, the magnitude of changes of CS parameters of those B atoms (B2, B13, and B15) which are directly bonded to diborinin ring are more in (c) model borininthan in (b) model.

Table 4 shows the average deviations of (b) model (and (c) model in bracket) for $\mathrm{CS}^{I}$ and $\mathrm{CS}^{\mathrm{A}}$ of ${ }^{11} \mathrm{~B}$ and ${ }^{15} \mathrm{~N}$ in all layers of DBD-BNNT. The positive average deviations show increasing the values of $\mathrm{CS}^{I}$ and $\mathrm{CS}^{A}$ in DBD-BNNT models relative to pure model. The changes of CS parameters of $\mathrm{B}$ atoms in the diborinin-doped (c) model are generally greater than (b) model. These orders are reverse for $\mathrm{N}$ atoms. 
TABLE 4: The average deviations of (b) model (and (c) model in brackets) for $\mathrm{CS}^{I}$ and $\mathrm{CS}^{A}$ of ${ }^{11} \mathrm{~B}$ and ${ }^{15} \mathrm{~N}$ in N-tip and B-tip of DBD-BNNT.

\begin{tabular}{|c|c|c|c|c|}
\hline & \multicolumn{2}{|c|}{$\mathrm{CS}^{I}$} & \multicolumn{2}{|c|}{$\mathrm{CS}^{A}$} \\
\hline & ${ }^{11} \mathrm{~B}$ & ${ }^{15} \mathrm{~N}$ & ${ }^{11} \mathrm{~B}$ & ${ }^{15} \mathrm{~N}$ \\
\hline Layer 1 (N-Tip) & $0.13[-2.00]$ & $-7.82[-4.93]$ & $5.47[5.12]$ & $8.68[5.56]$ \\
\hline Layer 2 & $-1.075[-0.95]$ & $-13.24[-10.42]$ & $1.1[-0.025]$ & $10.72[4.18]$ \\
\hline Layer 3 & $-1.16[-3.26]$ & $-8.85[-6.87]$ & $6.74[13.92]$ & $19.25[14.97]$ \\
\hline Layer 4 (B-Tip) & $0.05[2.03]$ & $-7.1[-9.25]$ & $-0.33[-1.77]$ & $10.32[16.25]$ \\
\hline
\end{tabular}

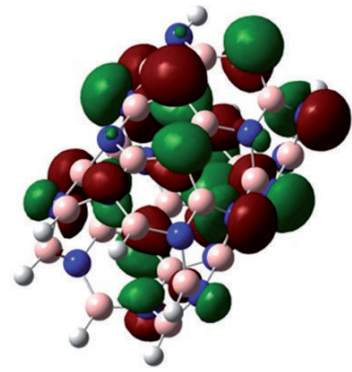

HOMO model

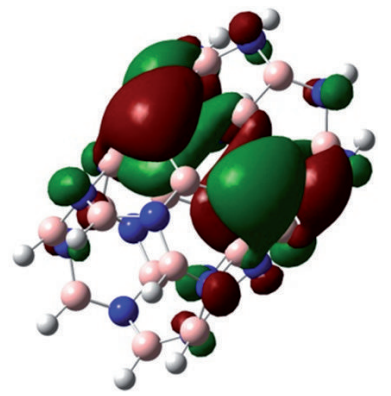

HOMO model

(b)

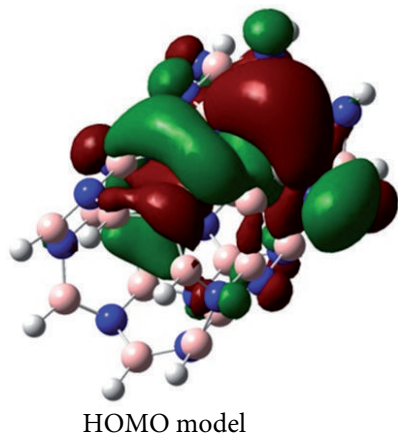

(c)

FIGURE 5: HOMO and LUMO orbitals for different models of the BNNTs; (a) pure model, (b) and (c) diborinin-doped models.

As a result, Table 4 shows changes in the average deviations of $\mathrm{N}$ atoms are greater than those of $\mathrm{B}$ atoms. Also changes of $C S^{I}$ and $\mathrm{CS}^{A}$ of ${ }^{11} \mathrm{~B}$ and ${ }^{15} \mathrm{~N}$ in layer 2 and 3 (directly bonded) are more than N-tip and B-tip of DBDBNNT.

\section{Conclusion}

In this study, we have used DFT calculations to investigate the properties of the electronic structure of pure and DBDBNNTs through to simulate the ${ }^{11} \mathrm{~B}$ and ${ }^{15} \mathrm{~N}$ spectra. The calculated structural energies yield similar values for the (b) and (c) models of DBD-BNNTs. However, the band gap differences between the forms (b) and the pure models are more than the differences between the forms (c) and the pure models of the BNNTs. Also, the B-N bond lengths and bond angles detect changes of diborinin-doped models. When diborinin is doped on the BNNT, dipole moment of the pure system is significantly changed. CS parameters detect the effects of diborinin-doping on the properties of nanotubes in comparison with the pure model. For the (b) and (c) models, both of the $\mathrm{CS}^{I}$ and $\mathrm{CS}^{A}$ parameters were significantly changed due to the diborinin-doped ring. The changes in the NMR $\left(\mathrm{CS}^{I}\right)$ parameters due to the diborinin doping are more significant for the $\mathrm{N}$ atoms than the $\mathrm{B}$ atoms regarding to the pure model.

Results show the Average deviations of (b) model [and (c) model in bracket] for $\mathrm{CS}^{I}$ and $\mathrm{CS}^{A}$ of ${ }^{11} \mathrm{~B}$ and ${ }^{15} \mathrm{~N}$ in all layers of DBD-BNNT. The positive average deviations show increasing the values of $\mathrm{CS}^{I}$ and $\mathrm{CS}^{A}$ in DBD-BNNT models relative to pure model. In the diborinin-doped (c) model, when compared to the (b) model, changes generally are greater in the values of CS parameters of those B atoms. These orders are reverse for $\mathrm{N}$ atoms.

Our results show that changes in the average deviations of $\mathrm{N}$ atoms are greater than those of $\mathrm{B}$ atoms. Also, changes of $\mathrm{CS}^{I}$ and $\mathrm{CS}^{A}$ of ${ }^{11} \mathrm{~B}$ and ${ }^{15} \mathrm{~N}$ in layer 2 , and 3 (directly bonded) are more than N-tip and B-tip of DBD-BNNT.

In DOS, the electronic structures observed near Fermi level of the pure BNNT show significant change due to the diborinin ring doping. So the band gaps near Fermi level become sharply narrower. It is clear that the doping of diborinin ring decreases the energy gaps of the pure BNNT.

For the pure model, the HOMO is located on the nitrogen atoms and the LUMO is uniformly distributed throughout the $\mathrm{B}-\mathrm{N}$ bonds. In contrast, for the diborinin-doped models, the majority of the HOMO and LUMO are located at the diborinin-doped regions.

\section{Acknowledgment}

This paper is dedicated to the beloved colleague Mr. Ali Karimian, who rests in peace at this time; but without his sincere assistance, this work would not be materialized. 


\section{References}

[1] S. Iijima, "Helical microtubules of graphitic carbon," Nature, vol. 354, no. 6348, pp. 56-58, 1991.

[2] J. Hu, T. W. Odom, and C. M. Lieber, "Chemistry and physics in one dimension: synthesis and properties of nanowires and nanotubes," Accounts of Chemical Research, vol. 32, no. 5, pp. 435-445, 1999.

[3] M. Law, J. Goldberger, and P. Yang, "Semiconducor nanotubes and nanowires," Annual Review of Materials Research, vol. 34, pp. 83-122, 2004.

[4] M. S. Gudiksen, L. J. Lauhon, J. Wang, D. C. Smith, and C. M. Lieber, "Growth of nanowire superlattice structures for nanoscale photonics and electronics," Nature, vol. 415, no. 6872, pp. 617-620, 2002.

[5] M. Farahani, T. S. Ahmadi, and A. Seif, "A DFT study of the nuclear magnetic response of the zigzag AlN-BN and BN-AlN nanotube junctions," Journal of Molecular Structure: THEOCHEM, vol. 913, no. 1-3, pp. 126-130, 2009.

[6] N. G. Chopra, R. J. Luyken, K. Cherrey et al., "Boron nitride nanotubes," Science, vol. 269, no. 5226, pp. 966-967, 1995.

[7] A. Rubio, J. L. Corkill, and M. L. Cohen, "Theory of graphitic boron nitride nanotubes," Physical Review B, vol. 49, no. 7, pp. 5081-5084, 1994.

[8] S. Riikonen, A. S. Foster, A. V. Krasheninnikov, and R. M. Nieminen, "Computational study of boron nitride nanotube synthesis: how catalyst morphology stabilizes the boron nitride bond," Physical Review B, vol. 80, no. 15, Article ID 155429, 14 pages, 2009.

[9] A. Zettl, J. Cumings, W. Han, and W. Mickelson, "Boron nitride nanotube peapods," AIP Conference Proceedings, vol. 633, pp. 140-144, 2002.

[10] R. Pati, P. Panigrahi, P. P. Pal, B. Akdim, and R. Pachter, "Gate field induced electronic current modulation in a single wall boron nitride nanotube: molecular scale field effect transistor," Chemical Physics Letters, vol. 482, no. 4-6, pp. 312-315, 2009.

[11] J. S. Lauret, R. Arenal, F. Ducastelle et al., "Optical transitions in single-wall boron nitride nanotubes," Physical Review Letters, vol. 94, no. 3, Article ID 037405, 4 pages, 2005.

[12] Y. Xiao, X. H. Yan, J. Xiang et al., "Specific heat of single-walled boron nitride nanotubes," Applied Physics Letters, vol. 84, no. 23, pp. 4626-4628, 2004.

[13] D. Jana, L. C. Chen, C. W. Chen, and K. H. Chen, "An abinitio approach to the optical properties of $\mathrm{CxNy}$ single wall nanotubes," Diamond and Related Materials, vol. 18, no. 5-8, pp. 1002-1005, 2009.

[14] R. J. Baierle, P. Piquini, T. M. Schmidt, and A. Fazzio, "Hydrogen adsorption on carbon-doped boron nitride nanotube," Journal of Physical Chemistry B, vol. 110, no. 42, pp. 21184-21188, 2006.

[15] F. Li, Y. Xia, M. Zhao et al., "Theoretical study of hydrogen atom adsorbed on carbon-doped BN nanotubes," Physics Letters A, vol. 357, no. 4-5, pp. 369-373, 2006.

[16] S. A. Shevlin and Z. X. Guo, "Hydrogen sorption in defective hexagonal BN sheets and BN nanotubes," Physical Review B, vol. 76, no. 2, Article ID 024104, 11 pages, 2007.

[17] E. Durgun, Y. R. Jang, and S. Ciraci, "Hydrogen storage capacity of Ti-doped boron-nitride and B Be-substituted carbon nanotubes," Physical Review B, vol. 76, no. 7, Article ID 073413, 4 pages, 2007.

[18] X. Wu, J. L. Yang, and X. C. Zeng, "Adsorption of hydrogen molecules on the platinum-doped boron nitride nanotubes,"
Journal of Chemical Physics, vol. 125, no. 4, Article ID 044704, 6 pages, 2006.

[19] M. J. Frisch, G. W. Trucks, H. B. Schlegel et al., GAUSSIAN 98, Gaussian, Inc., Pittsburgh, Pa, USA, 1998.

[20] R. G. Pearson, Hard and Soft Acid and Bases, Dowden, Hutchinson and Ross, Stroudsberg, Pa, USA, 1973.

[21] R. G. Pearson, "Hard and soft acids and bases-the evolution of a chemical concept," Coordination Chemistry Reviews, vol. 100, pp. 403-425, 1990.

[22] R. G. Pearson, Chemical Hardness, Wiley-VCH, Oxford, UK, 1997.

[23] P. W. Ayers and R. Parr, "Variational principles for describing chemical reactions: the Fukui function and chemical hardness revisited," Journal of the American Chemical Society, vol. 122, no. 9, pp. 2010-2018, 2000.

[24] L. Pauling, The Nature of Chemical Bond, Cornell University Press, New York, NY, USA, 1960.

[25] K. D. Sen and C. K. Jorgensen, Electronegativity: Structure and Bonding, vol. 66, Springer, Berlin, Germany, 1987.

[26] I. Alkorta and J. Elguero, "Computational NMR spectroscopy," in Computational Spectroscopy: Methods, Experiments and Applications, J. Grunenberg, Ed., Wiley-VCH, Weinheim, Germany, 2010.

[27] M. Mirzaei and M. Miirzaei, "An electronic structure study of O-terminated zigzag BN nanotubes: density functional calculations of the quadrupole coupling constants," Solid State Communications, vol. 150, no. 27-28, pp. 1238-1240, 2010.

[28] M. Mirzaei, "The NMR parameters of the SiC-doped BN nanotubes: a DFT study," Physica E, vol. 42, no. 7, pp. 1954-1957, 2010.

[29] M. Mirzaei, "Calculation of chemical shielding in C-doped zigzag BN nanotubes," Monatshefte fur Chemie, vol. 140, no. 11, pp. 1275-1278, 2009. 

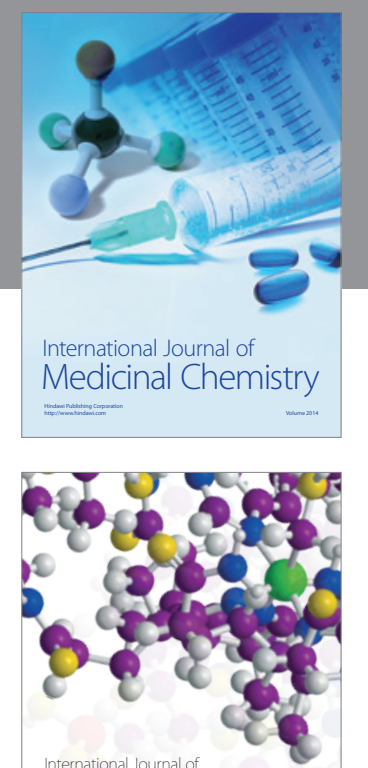

\section{Carbohydrate} Chemistry

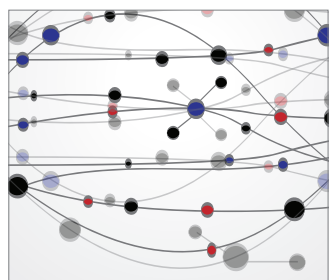

The Scientific World Journal
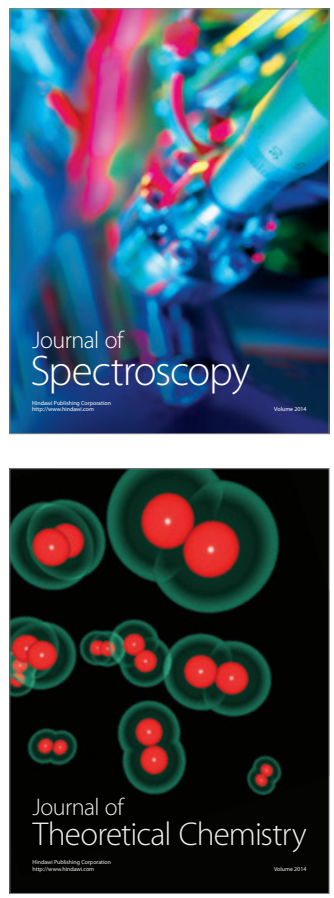
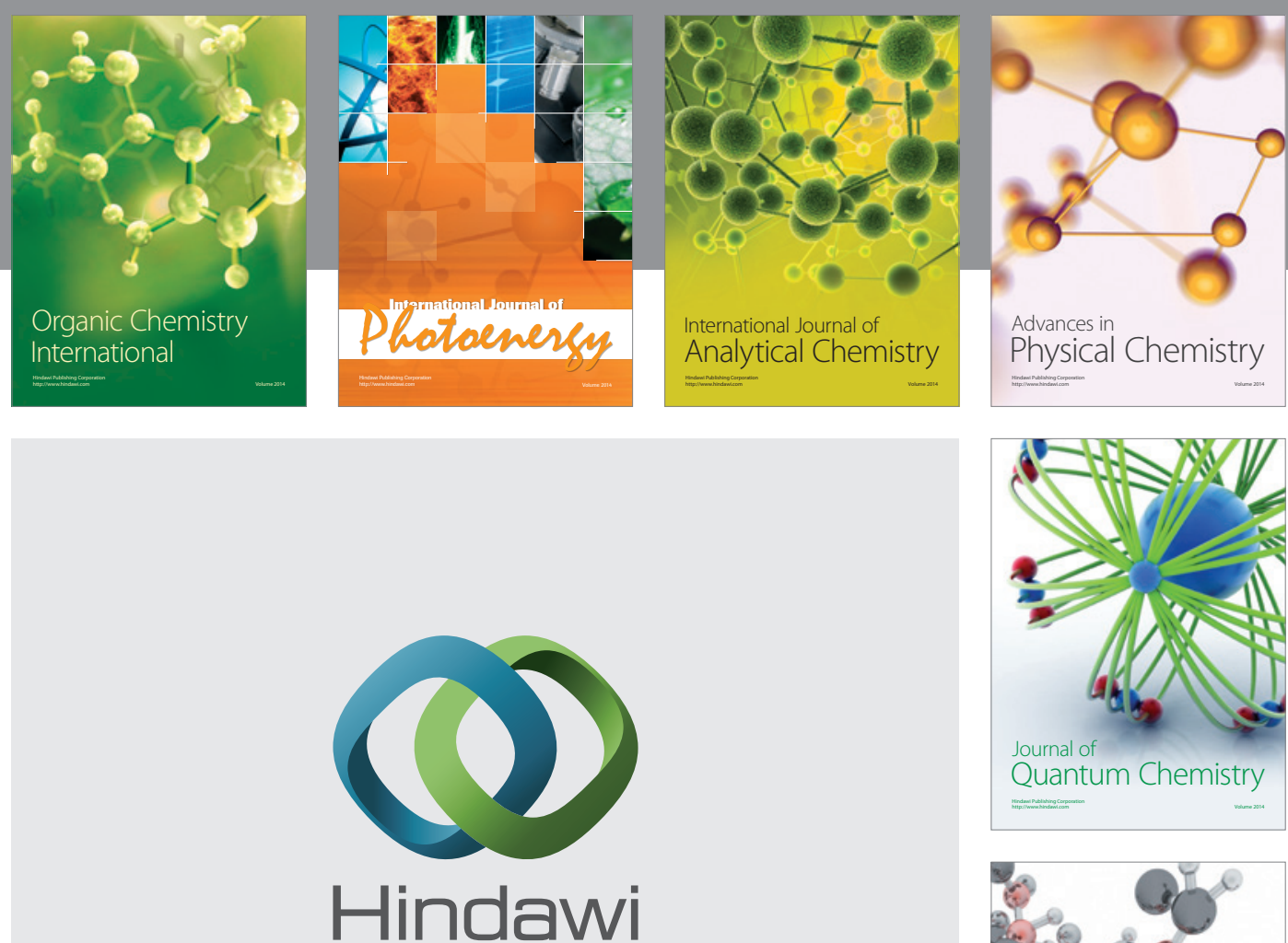

Submit your manuscripts at

http://www.hindawi.com

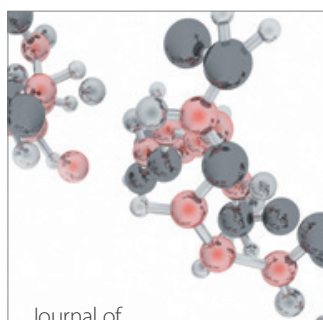

Analytical Methods

in Chemistry

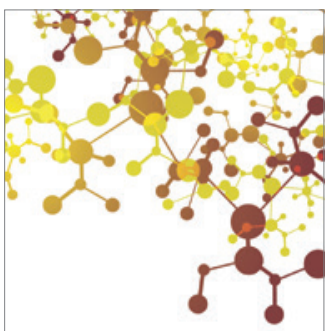

Journal of

Applied Chemistry

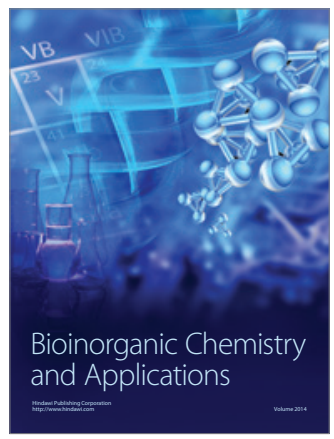

Inorganic Chemistry
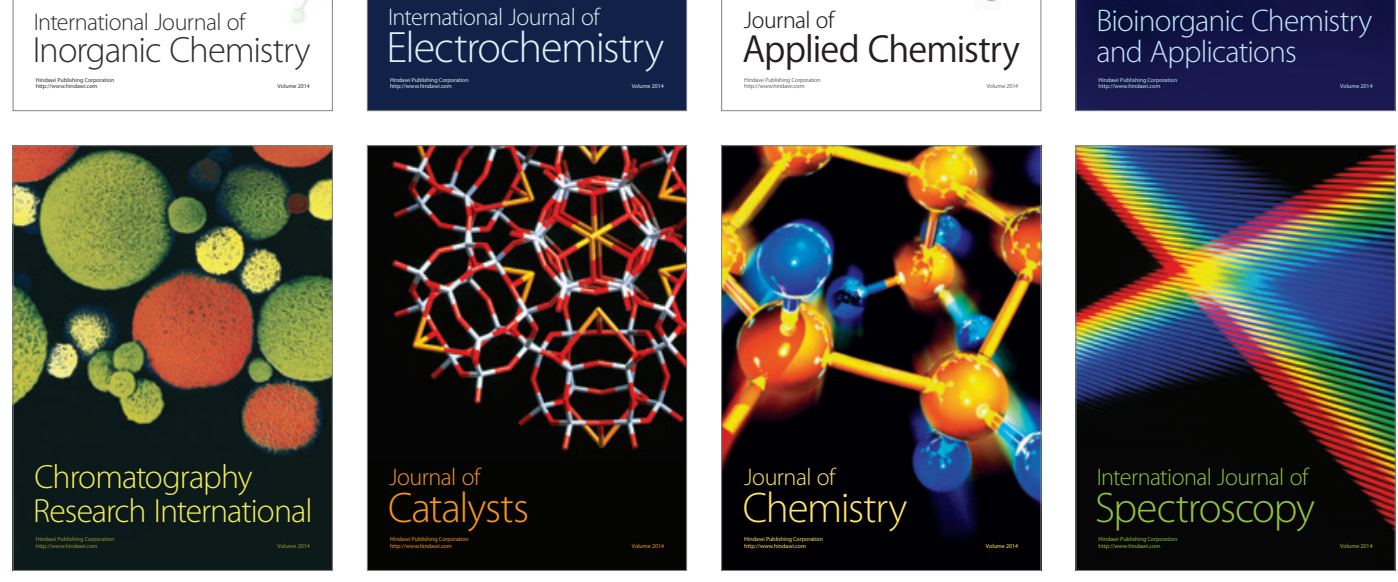